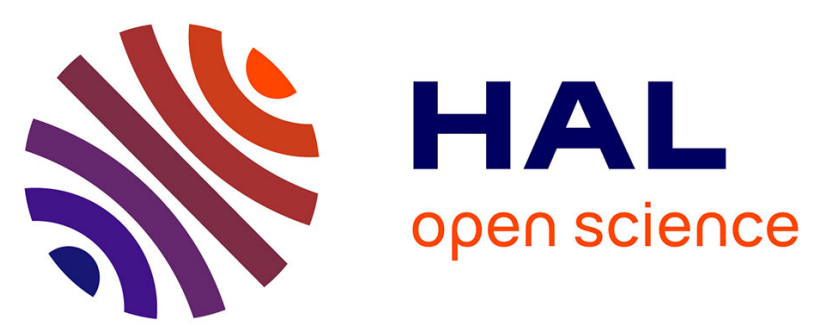

\title{
Characterization of time-varying regimes in remote sensing time series: application to the forecasting of satellite-derived suspended matter concentrations
}

Bertrand Saulquin, Ronan Fablet, Pierre Ailliot, Grégoire Mercier, David Doxaran, Antoine Mangin, Odile Fanton d'Andon

\section{To cite this version:}

Bertrand Saulquin, Ronan Fablet, Pierre Ailliot, Grégoire Mercier, David Doxaran, et al.. Characterization of time-varying regimes in remote sensing time series: application to the forecasting of satellite-derived suspended matter concentrations. IEEE Journal of Selected Topics in Applied Earth Observations and Remote Sensing, 2014, 8 (1), pp.406 - 417. 10.1109/JSTARS.2014.2360239 . hal01188636

\section{HAL Id: hal-01188636 \\ https://hal.science/hal-01188636}

Submitted on 12 Oct 2015

HAL is a multi-disciplinary open access archive for the deposit and dissemination of scientific research documents, whether they are published or not. The documents may come from teaching and research institutions in France or abroad, or from public or private research centers.
L'archive ouverte pluridisciplinaire HAL, est destinée au dépôt et à la diffusion de documents scientifiques de niveau recherche, publiés ou non, émanant des établissements d'enseignement et de recherche français ou étrangers, des laboratoires publics ou privés. 

series: application to the forecasting of satellite-derived suspended matter concentrations

Doxaran (4), Antoine Mangin (2), Odile Fanton d'Andon (2).

(1) ACRI-ST, Sophia-Antipolis, 260 route du Pin Montard, BP 234

706904 Sophia-Antipolis, France

8 (2) Institut Mines-Telecom, Télécom Bretagne; UMR CNRS 3192 Lab-STICC, Technopôle

9 Brest Iroise CS 83818, 29238 Brest, France

10 (3) Université Européenne de Bretagne, 35000 Rennes, France

11 (4) CNRS, Université Pierre et Marie Curie-Paris 6, UMR 7093, Laboratoire d'Océanographie

12 de Villefranche/Mer, 06230 Villefranche-sur-Mer, France

13 Corresponding author: bertrand.saulquin@acri-st.fr

14 Submission date: $19 / 05 / 2014$

\section{Abstract}

Satellite data, with their spatial and temporal coverage, are particularly well suited for the analysis and characterization of space-time-varying relationships between geophysical processes. In this study, we investigate the forecasting of a geophysical variable using both satellite observations and model outputs. The studied latent-regime models aim here at identifying time-varying regime 
21 shifts within a dataset which is a key of interest for geophysical processes driven by the seasonal

22 variability. As a specific example, we study the daily concentration from 2007 to 2009 of mineral suspended particulate matters estimated from the satellite-derived MODIS, MERIS and SeaWiFS dataset, in coastal waters adjacent to the Gironde River mouth (South West of France). We clearly show that the forecast of the high resolution suspended particulate matter dataset using environmental data (wave height, wind strength and direction, tides and river outflow) and a multiregime model is significantly improved compared with a classical multi-regression and a Support

28 Vector Regression model. Each regime is here characterized by a regression function and a covariance structure.

From an analytical point of view, we compare the results obtained with four models:

31 homogeneous and non-homogeneous Markov-switching models, with and without an autoregressive term, i.e. the suspended matter concentration observed the day before. Inclusion of an autoregressive term is motivated by the strong natural autocorrelation level depicted by geophysical time series, but, one may avoid this term if, for example, the observations are no more available during specific conditions or periods. With the evaluated models, best results are obtained with a mixture of 3 regimes for both autoregressive and non-autoregressive models. Prediction performance at day +1 , using the non-autoregressive models and a validation dataset, reached $80 \%$ of the observed variance, compared to $32 \%$ for a standard single-regime (regression) analysis, and $40 \%$ for a Support Vector Regression. Inclusion of an autoregressive term increases results to $93 \%$ of explained variance for the mixture model compared to $80 \%$ without autoregressive term and $85 \%$ using a Support Vector Regression. These results stress the potential of the identification of geophysical regimes to improve the forecasting, or the inversion, of a high resolution geophysical variable using both observations and model outputs. We also show that for short periods of lack of observations (less than 15 days), estimations using the autoregressive term are better than without. 


\section{1}

Index term: 1) Satellite-derived suspended matter time series analysis. 2) Statistical forecasting. 3) Regime-switching latent regression models. 4) Joint analysis of satellite-derived products and operational model outputs. 5) Gironde river plume.

\section{Introduction}

The forecasting of a geophysical variable using statistical models is an alternative to modelbased approaches which typically involve complex simulation and/or assimilation [1, 2]. For instance, coupled hydrodynamic and sediment transport models can be used to estimate the concentration of suspended particulate matters within the water column [3] while statistical approaches may use available satellite and model data to predict the same variable [4]. Many statistical approaches have been proposed and evaluated to forecast or infer a studied variable from predictors. Among them, linear multivariate regression [5] and non-linear (polynomial) multivariate regression [6] are the most known. Numerous specific models dedicated to time-series analysis such as AutoRegressive Moving Average (ARMA) and AutoRegressive Integrated Moving Average (ARIMA) models [7] have also been developed initially to address financial time series. These latest, which aim at studying the behavior of a time series without considering forcing factors, have 3 also been applied to geophysical time series [8]. Non-linear regressions, based on supervised learning strategies, such as Neural Networks [9] and Support Vector Regressions (SVR) [10] may provide relevant alternatives to estimate a variable from predictors. In the context of geophysical studies, they may nevertheless suffer from two major drawbacks. First, though relevant regression performances may be reported, these models are not physically interpretable and may be very sensitive to the training dataset. Second, multi-regime dynamics, often exhibited by geophysical processes driven by the seasonality [11], cannot be addressed by non-linear models, contrary to latent-regime models as demonstrated in our study. 
71 We propose here to characterize time-varying relationships between a variable and its forcing 72 parameters using latent-regime models, and hence optimize forecasting results. As an illustration, 73 we address the concentration of inorganic suspended particle matters (SPIM), estimated from satellite data using a regional algorithm $[12,13]$, and observed in the mouth of the Gironde estuary. In this area, sediments are mainly exported from the Gironde estuary [13, 14] and SPIM concentration clearly depends on the local physical forcing: swell, tide, wind and river discharge. A minimum of energy has to be brought by waves and tides to re-suspend cohesive sediments accumulated at the bottom. Conversely, when sediments have been re-suspended in the water column by wave influence, their settling velocity depends on their size and density [15] and physico-chemical properties [16]. This example stresses that the relationships between the studied

81 variable (SPIM) and the causing factors evolve in space and time and potentially requires advanced statistical methods to identify the underlying geophysical regimes.

From a methodological point of view, "latent regime regressions" also referred as "clusterwise regressions" [17, 18] are particularly appealing to identify such non-linear and multi-regime patterns within a dataset. Each regime is associated with a linear regression and a non-linear

86 relationship is thus estimated as a sum of linear contributions. Regarding the temporal dynamics of 87 these regimes, we here consider Markovian processes [19], which state the transitions in time 88 between two regimes. The standard Hidden Markov Model (HMM) and Non-Homogeneous 89 Markov Model (NHMM) are evaluated [19]. The inclusion of an autoregressive term (HMM-AR) and (NHMM-AR) is also discussed. This aspect is motivated on the one hand by the strong 91 autocorrelation level depicted by geophysical time series [20]. When the observation at $\mathrm{t}-1$ is available, it is obvious, considering the strong natural autocorrelation of geophysical data that the

93 forecast at time $\mathrm{t}$ should take into account the observation at time $\mathrm{t}-1$. Conversely, for specific 94 applications, or if the observations are not available during long periods (such as winter storms, or 
previous days. We discuss here the choice between autoregressive or not autoregressive models for long lack of observation period using forecasting results from $t+1$ to $t+15$. points in the mouth of the Gironde estuary in the $\left[3^{\circ} \mathrm{W}-1^{\circ} \mathrm{E} ; 45-46.5^{\circ} \mathrm{N}\right]$ area during the period 100 2007-2009. Validation is performed on the same area for using the data for the year 2010. We used 101 EOFs to reduce the dimension of the space-time observations. This is a usual approach in spatio102 temporal statistics $[21,22]$ although alternatives may be considered such as linear discriminant 103 analysis [23], and, we could also introduce a latent variable to describe the regime at each location 104 and interact with the regimes at other locations. Nevertheless, such models are known to be very 105 difficult to fit on the data and remain a research challenge for statisticians. We infer our mixture 106 model using the expansion coefficients of the first four modes of the EOF which explain $99 \%$ of the 107 total variance. The variables used as predictors for the SPIM expansion coefficients (EC) are the 108 wave height issued from a numerical model [24], the wind fields optimally interpolated from 109 satellite observations [25], the tide coefficient [26] and the Gironde fresh water discharge (sum of 110 the Garonne and Dordogne Rivers contributions).

\section{Methods}

\section{$112 \quad 2.1$ Markov switching forecast models}

We address here the study of a two dimensional scalar geophysical time series Y. In a hidden

115 Markov model framework (HMM; [19]), one states two different processes, the observed process Y

116 and a hidden process $\mathrm{Z}$. The observed process (here the turbidity) is assumed to be temporally 117 dependent of the hidden process. The hidden process $Z_{t}$ is modeled as a first order Markov chain 118 [19]. At a given time $t$, the hidden variable $Z_{t}=k$ is a discrete value which states the regime 
119 characterized by a latent [17] regression model with coefficient $B_{k}$ between the variable $Y_{t}$ and the 120 predictor $\mathrm{X}_{\mathrm{t}}$. At time $\mathrm{t}$, knowing regime variable $\mathrm{Z}_{\mathrm{t}}$, the observed variable $\mathrm{Y}_{\mathrm{t}}$ is modeled as:

where $X t B k$ is the regression function, which predicts variable $Y_{t}$ from some predictors $X_{t}$ for

123 regime $\mathrm{Z}_{\mathrm{t}}=\mathrm{k}$.

Figure 1 shows a graphical representation of the conditional dependencies involved in the model, in the form of the general Directed Acyclic Diagram (DAG). It illustrates the interactions between the variable $Y_{t}$, the predictors $X_{t}$, the hidden regime $Z_{t}$ and the covariate $S_{t}$ which acts on regime switching. $X_{t}$ may contain lagged values of $Y_{t}$ (referred as autoregressive terms) and/or exogenous covariates such as wind or wave height. Figure 1 defines a general family of model

129 which encompasses the most usual ones with regime switching. When no covariate is considered 130 i.e., $Z_{t}$ only depends $Z_{t-1}$, and, $Y_{t}$, only depends on $\left(Y_{t,-s} . . Y_{t,-1}\right)$ and $Z_{t}$, we retrieve the usual Markov 131 switching autoregressive (MS-AR). If we further assume that $\mathrm{s}=0$ (without autoregressive 132 component where $\mathrm{Y}_{\mathrm{t}}$, only depends on $\mathrm{Z}_{\mathrm{t}}$ ) then we obtain the Hidden Markov Models (HMMs). 133 When $\mathrm{Z}_{\mathrm{t}}$ does not dependent on $\mathrm{Z}_{\mathrm{t}-1}$, and the dependence on $\mathrm{S}_{\mathrm{t}}$ is parameterized using indicator 134 functions, we obtain the threshold autoregressive (TAR) model which is the other important family 135 with regime switches in the literature. HMMs, MS-AR and TAR have been used in many fields of applications including geosciences [27]. 


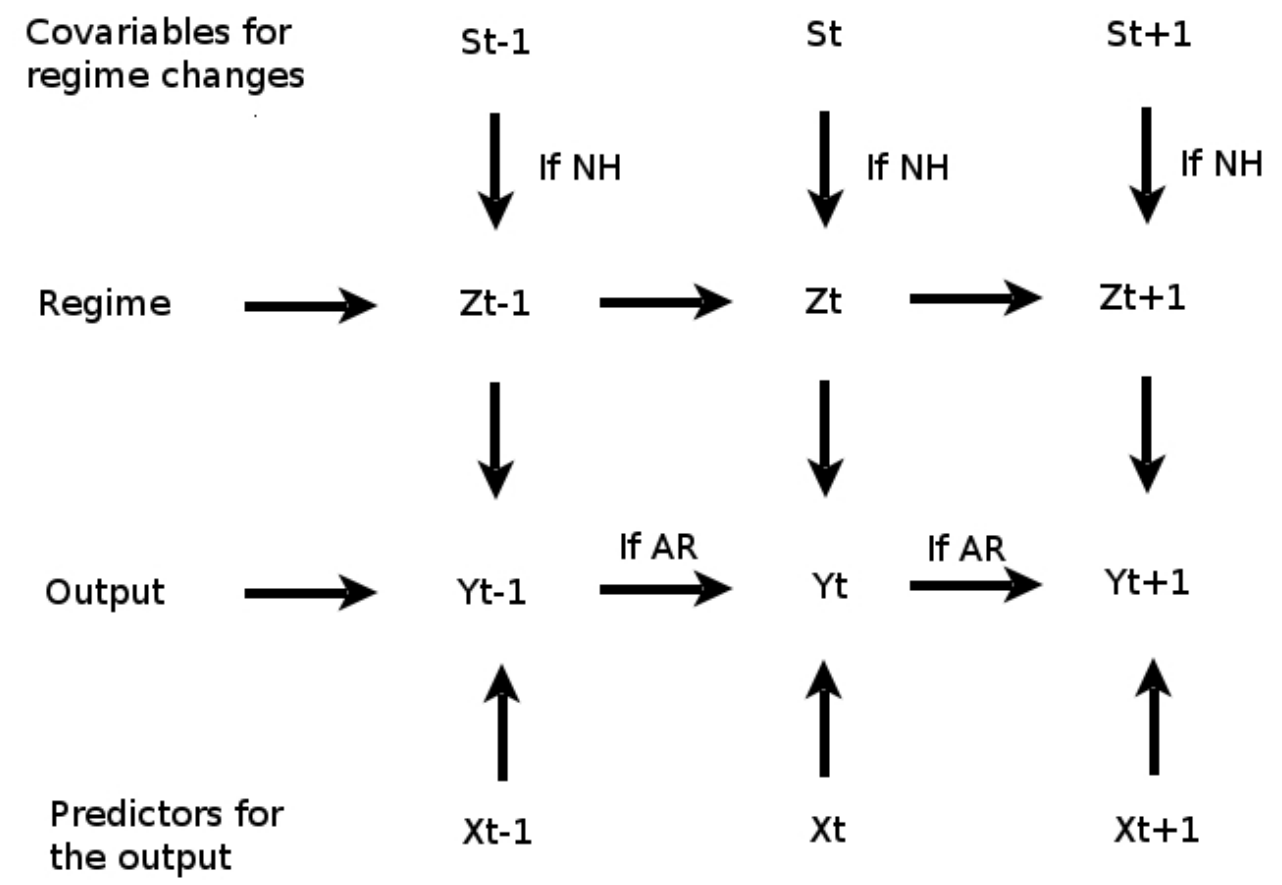

Figure 1: Graphical representation of the various Markov-Switching Models considered in this 139 work: the arrows state the conditional dependencies between the random processes in play, namely 140 hidden regime process $\mathrm{Z}$, observed process $\mathrm{Y}$, prediction process $\mathrm{X}$ and regime change covariate process $\mathrm{S}$.

In the following equations (2 to 14), $\mathrm{X}_{\mathrm{t}}$ and $\mathrm{S}_{\mathrm{t}}$ are known, as they are either observations or 143 numerical model outputs. Given (1) the conditional likelihood of the observation $\mathrm{Y}_{\mathrm{t}}$ given 144 predictors $X_{t}$ and regime $Z_{t}$ is expressed as [17]:

146 where $\mathrm{N}$ represents the Gaussian probability density function with mean XtBk and covariance $147 \sigma_{\mathrm{k}}$. Hence, given predictors up to time $\mathrm{t}$ we can predict process $\mathrm{Y}$ from its expectation conditionally 148 to process $\mathrm{X}$ : 


\section{Markov-switching priors}

Stating hidden regime process $\mathrm{Z}$ as a first-order Markovian process amounts to modeling the transition between successive regimes at time $\mathrm{t}$ and time $\mathrm{t}-1$. In the simplest case, one assumes homogeneous transitions, i.e. time-independent and context-independent transitions, and the

Markovian process is fully characterized by its transition matrix $\mathrm{P}\left(\mathrm{Z}_{\mathrm{t}}=\mathrm{k} \mid \mathrm{Z}_{\mathrm{t}-1}=\mathrm{l}\right)$ for possible pairs of successive regimes $(\mathrm{k}, 1)$. In the HMM setting the conditional distribution of $Z_{t}$, given the past values $Y_{s}$ and $Z_{s}$ for $s<t$, is assumed to depend only on $Z_{t-1}$ (Fig. 1):

$$
\pi t k=P Z t=k Y 0 . . Y \mathrm{t})=l=1 \mathrm{KPYt} Z t=k \cdot P(Z t=k Z t-1=l . P Z t-1=l Y 0 . . Y \mathrm{t}-1)
$$

A NHMM extends this idea by allowing the transition matrix between the hidden states to depend on a set of observed covariates $S_{t}$. Hughes and Guttorp $[28,29]$ highlighted the added value of the NHMM to characterize the links between the large-scale atmospheric measures and the small-scale spatially discontinuous precipitation field. In the NHMM settings, the transition matrix between states $P\left(Z t=k Z t-1=l\right.$ in (3) is now time-dependent and conditioned by the covariates $\mathrm{S}_{\mathrm{t}}$ :

$$
\pi t k=P Z t=k Y 0, . ., Y \mathrm{t}, \mathrm{St})=
$$

$$
l=1 K P Y t \quad Z t=k, . P(Z t=k Z t-1=l \text {, St.PZt-1=l YO ..Yt }-1)
$$



$S_{t}$ given the state transition $\left(Z_{t}, Z_{t+1}\right)$. We suppose that the probability density function of the covariates during this change of state follows a normal distribution: gaussian distribution $\Sigma_{1, \mathrm{k}}$ is a covariance matrix. In the present application, and to reduce the number of parameters to be estimated, we consider that the predictors are uncorrelated (null covariance) and their relative influence is identical (same variance), i.e. $\Sigma_{1, \mathrm{k}}$ is a multiple of the identity matrix.

\subsection{Estimation of the model parameters}

The considered models involve two categories of parameters: those of the observation model, $\theta_{\mathrm{k}}$ namely regression coefficient $B_{k}$ and standard deviation $\sigma_{k}$ for each regime (Eq. 2) and those of the Markov-swtiching prior, namely $\theta_{\mathrm{s}}$ (Eq. 5). Given observed $\mathrm{Y}$ and $\mathrm{X}$ series, we proceed to the estimation of model parameters according to a classical maximum likelihood (ML) criterion using an iterative Expectation Maximisation (EM) procedure [30] expressed here without covariates: 
where $\theta=\left\{\theta_{\mathrm{s}}, \theta_{\mathrm{k}}\right\}$ is the set of parameters to be estimated. For a given initialization for the

191 parameters the EM procedure iterates estimation steps (E-step) of the posterior regime likelihood $192 \pi t k$ with the given modes and the maximisation step (M-step) for the update of the parameters given 193 these posteriors. The algorithm iterates until convergence between steps $\mathrm{n}$ and $\mathrm{n}+1$, i.e. $194|L \theta(n)-L \theta(n+1)|<10-3$. The posterior likelihood $\pi t k \quad(\mathrm{Eq} 3 \& 4)$ of the latent regime $Z_{\mathrm{t}}$, is 195 estimated in the E-step using the classical forward-backward recursions [31, 32] given series $\mathrm{X}$ and $196 \mathrm{Y}$ and current parameter estimate $\theta_{\mathrm{k}}^{(\mathrm{n})}, \theta_{\mathrm{s}}^{(\mathrm{n})}$. The M-step re-estimates the parameters $\theta_{\mathrm{k}}^{(\mathrm{n}+1)}, \theta_{\mathrm{s}}^{(\mathrm{k}+1)}$. 197 For this, it is often possible to break the optimization problem into several lower dimensional 198 optimization problems which are much quicker to solve [32]. More precisely, for all the models 199 considered in this paper, it is possible to separate the parameters related to the evolution of the 200 hidden Markov chain $\theta_{\mathrm{s}}$, and the parameters related to the evolution of the observed process in each 201 regime $\theta_{\mathrm{k}}$ : $\theta=\operatorname{argmax} \theta s t \log (P(Z t=k Z t-1=l, \mathrm{St}, \theta s(\mathrm{n})) P Z t=k, Z t-1=l \mathrm{Y} 0 . . \mathrm{YT}, \mathrm{St}, \theta(\mathrm{n})$

\subsection{Forecasting application}

The considered multi-regime regression models are applied to the short-term forecasting of series $\mathrm{Y}$. More precisely, at a given time $\mathrm{t}$, we aim at predicting variable $\mathrm{Y}$ at time $\mathrm{t}+\mathrm{dt}$. We

210 typically assume that prediction variables $\mathrm{X}$ and covariates $\mathrm{S}$, typically numerical simulations, are 211 available up to time $\mathrm{t}+\mathrm{dt}$ whereas the variable $\mathrm{Y}$ is only known up to time $\mathrm{t}$. is estimated using $212 \mathrm{X}_{0 . \mathrm{t}+\mathrm{dt}}$ and $\mathrm{S}_{0 . .+\mathrm{dt}}$ (for inhomogeneous transitions). Thus, $\mathrm{Y} t+\mathrm{dt}$ is given by the conditional 
213 expectation of variable $\mathrm{Y}_{\mathrm{t}+\mathrm{dt}}$ given observations series up to time $\mathrm{t}$ and predictor series up to time $\mathrm{t}+$ $214 \mathrm{dt}:$

216 For HMM and NHMM it resorts to:

218 For autoregressive models HMM-AR and NHMM-AR, i.e. $X_{t+d t}$ contains $Y_{t+d t-1}$ which is not 219 available, is estimated using $\mathrm{Y}_{\mathrm{t} . .}, \mathrm{X}_{\mathrm{t}} . . \mathrm{X}_{\mathrm{t}+\mathrm{dt}-1}$ and . Estimated $\mathrm{Y} t+\mathrm{dt}$ resorts to:

221 It might be noted that these predictions actually account for the uncertainties in the 222 determination of the underlying regimes. Contrary to deterministic methods, confidence interval 223 and uncertainties on $\mathrm{Y} t+\mathrm{dt}$ can be derived [33] which is a key issue for modeling considerations.

\subsection{Model performance estimation}

A key issue in practice, which has received lots of attention in the last few years, is the problem 228 of model selection which aims at finding the "optimal" number of predictors and covariates [31]. 229 Hereafter, we have chosen to use both the Bayes Information Criterion (BIC) and the explained 230 variance (EVAR) as a first guides. BIC index generally permits to select parsimonious models 231 which fit the data well [34]. It is defined as: 
Where $\mathrm{L}$ is the likelihood of the data, $\mathrm{p}$ is the number of parameters and $\mathrm{S}$ is the number of 234 observations. The likelihood which is an output of the backward-forward recursions performed in 235 the E-step. We also use the classical explained variance, EVAR, to characterize the model 236 relevance:

$$
\text { EVAR=1. }-\operatorname{var} \mathrm{Y} t+1-\mathrm{Y} t+1 / \operatorname{var}(\mathrm{Y} t+1)
$$

BIC and EVAR are partially linked [34]. BIC tends to penalize complex models whereas explained variance criterion only qualifies the result and may lead to the over-parameterization of a model that typically lead to errors when other dataset are tested using the same parameterization.

241 Therefore we consider both BIC and EVAR to assess the model performance. predictors the variable showing a significant correlation with the studied variable. Given these predictor datasets, we tested all the possible configurations and chose the predictors which provide the lower BIC on the training dataset and the greatest EVAR using the training (EVAR_train) and the validation dataset (EVAR_valid).

\section{The data}

\subsection{The studied variable}

Non-algal SPM concentrations (SPIM) are estimated using an analytical algorithm [12] defined as the difference between total SPM and phytoplankton biomass, the latter derived from Chl-a. It

254 incorporates mainly mineral SPM and smaller amounts of organic SPM not related to living 
phytoplankton. This method to derive non-algal SPM from remote-sensing reflectance is based on the inversion of a simplified equation of radiative transfer, assuming that chlorophyll concentration is known. This merged dataset consists of fields of non-algal surface SPM concentrations, derived from the Sea-viewing Wide Field-of-view Sensor (SeaWiFS), the Moderate Resolution Imaging Spectroradiometer (MODIS) and the Medium Resolution Imaging Spectrometer (MERIS) sensors, provided by the Ocean Colour TAC (Thematic Application Facility) of MyOcean, and interpolated with a kriging method [35] for the period 2007-2009 over the Gironde mouth river from $3^{\circ} \mathrm{W}-1^{\circ} \mathrm{E}$; $45-46.5^{\circ}$ N. Finally 5682 continuous time series of 1096 days compose our initial dataset of mineral suspended matters concentration.

We first account for the space-time variability of the dataset, previously detrended and centered for each time series [37] using a EOF decomposition [21], expressed here using the matrix form:

where $U$ is a here $5682 * 5682$ matrix containing the spatial modes (Eigenvectors) of the covariance decomposition (ordered by percentage of explained variance). Associated with each spatial mode $\mathrm{k}$, its expansion coefficient (also referred in the literature as principal component) is the time evolution of the $\mathrm{k}^{\text {th }}$ mode:

$$
\text { EC_SPIM }{ }_{k, t}=\operatorname{SPIM}_{\mathrm{t}} * \mathrm{U}_{\mathrm{k}}
$$

Figure 2 shows the four first spatial modes of the EOF decomposition. Figure 3 depicts the four associated time series EC_SPIM $\mathrm{i}_{\mathrm{i}, 4,4}$. The first mode (Fig.2a) comprises $85 \%$ of the total variance. It clearly addresses the seasonal cycle as shown in Fig. 3a where the switch between winter (high values of EC_SPIM 1 correspond here to high values of SPIM observed in winter) and summer periods is clearly visible. The variability around the seasonal mean is captured by the other modes (Fig.2 c-e \& Fig 3 c-e). Mode 2 refers to the inter-annual and the intra-seasonal variability in the 
gradients and represents $4 \%$ of the total variance and mode 4 is clearly influenced by the Gironde 280 river (Fig. 2d), which brings sediments during water outflow, and represent $3 \%$ of the variance. By 281 construction, EOF decomposition imposes the orthogonality [21] of the spatial modes (Fig. 2).
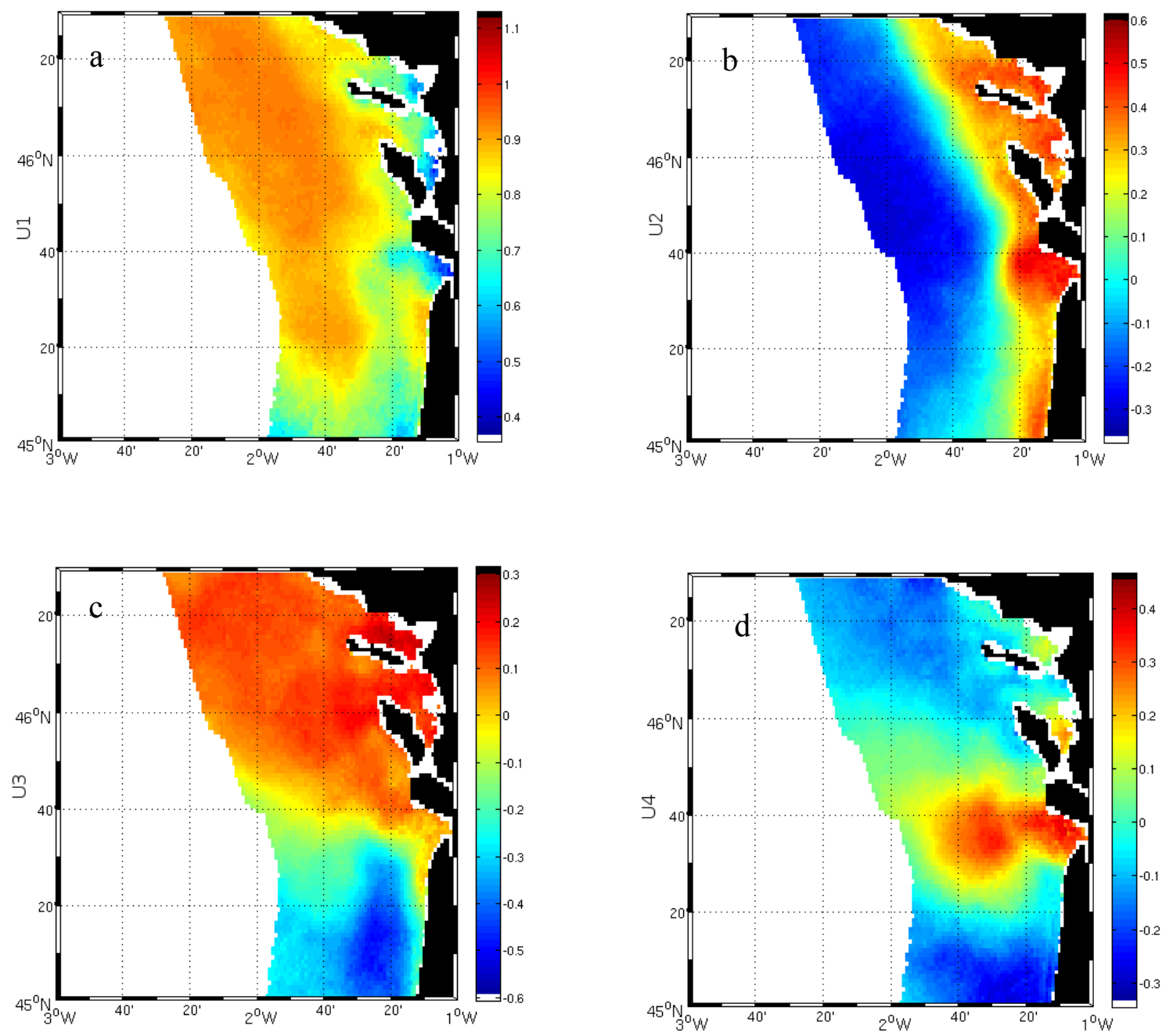

Figure 2: spatial modes of the EOF decomposition of the SPIM observed from satellite from 283 2007-2009 in the Gironde mouth river. From left to right and top to bottom the first four EOF 284 modes account respectively for $85,7,4$ and $3 \%$ of the total variance. 

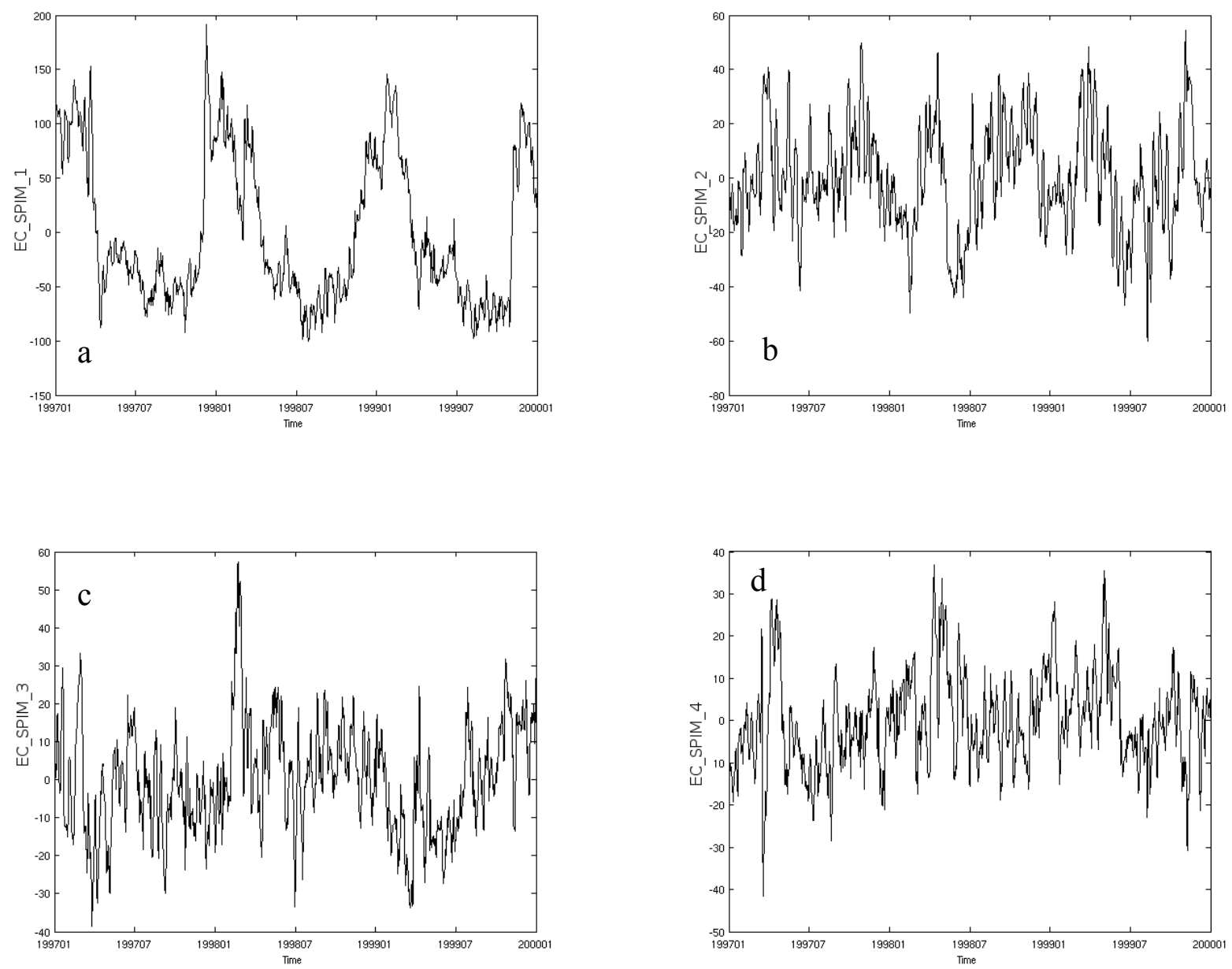

Figure 3: EOF decomposition of the SPIM observed from satellite from 2007-2009 in the

286 Gironde mouth river: from left to right and top to bottom, the expansion coefficients (EC_SPIM $\left.{ }_{1-4}\right)$ 287 associated with the first four EOF modes depicted in Fig. 2, i.e. the time evolution of the spatial 288 modes. The reconstruction of the SPIM variable from the estimated ECs is performed as:

The total explained variance using the 4 first modes is shown Fig. 4. On average, the explained 291 variance represents $99 \%$ of the total variability on the areas with some local minima of $60 \%$ 292 observed at the very near-shore and the Southwestern part of the area. 


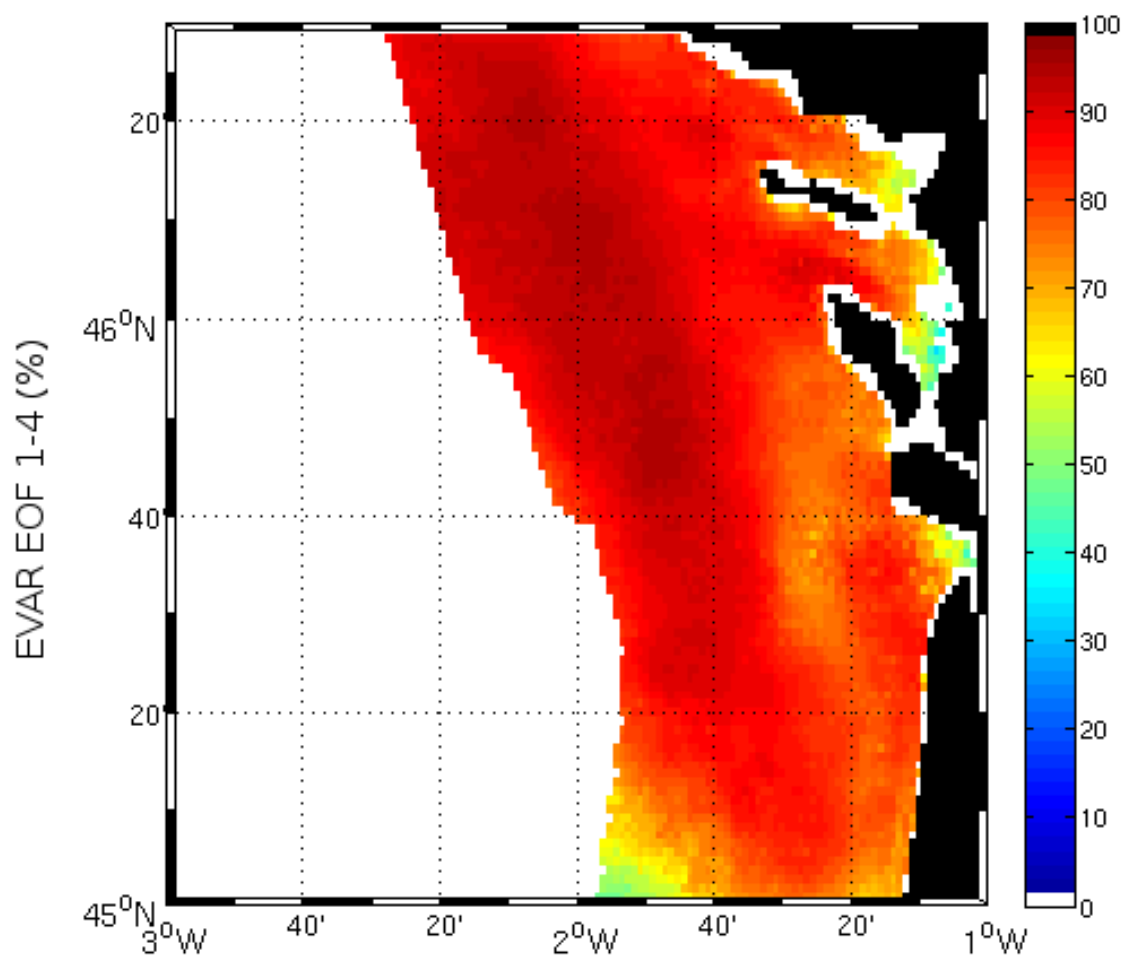

Figure 4: Variance explained by the four first modes of the EOF decomposition of the suspended matters.

\subsection{Predictors and covariates}

The predictors $\mathrm{X}$ are the variables used in the estimation of $\mathrm{Y}$ and $\mathrm{Z}$ any time (Eq. $13 \& 14$ ). We used here wave height (WH) daily means of the Wave Watch 3 model (WW3; [24, 36]) provided by

301 the IOWAGA and PREVIMER programs, Western and Northern winds interpolated from QuickSCAT and ASCAT observations in conjunction with ECMWF forecasting [25], provided by Ifremer, tide index (SHOM, 2000) at Bordeaux and the flow measurement of river la Gironde. Similarly to the SPIM data, all the data were log transformed. For the wind data which is signed, the transformed log variable was signed negatively a posteriori to the log transformation. The WH first mode of the EOF decomposition explained $98 \%$ of the total variance, $93 \%$ for the Northern wind 
(WND1), and 96\% for the Western wind (WND2). Covariates are the normalized predictors used in

308 the estimation but considered at $\mathrm{t}-2$. This lag has been estimated as the optimal time-lag using BIC and EVAR results on the training dataset.

\section{Results}

We summarize in Table 1 the prediction performance for the first four ECs of the SPIM issued

314 from four models: HMM, NHMM, HMM-AR, and NHMM-AR. The number of considered modes

315 for the mixture varies from 1 to 3 . The one-mode models refer to a simple multivariate regression 316 analysis. For each configuration we provide the BIC and EVAR_train on the training dataset 317 (2007-2009) and EVAR_valid on the validation dataset (2010). Note that the selection of the 318 predictors and resulting covariates is achieved as a prior step as described in Section 2.6. The first 319 mode of the EOF decomposition explains $85 \%$ of the total variance. EC_WH $\mathrm{WH}_{1}$ and EC_WND2 1 320 (respectively the expansion coefficient of the first EOF of the Western winds) are identified as 321 being the relevant predictors. This mode captures the mean seasonal variability of the SPIM, which 322 is mainly driven by WH and the North Atlantic storms and at a second order by the Western winds. 323 For EC_SPIM 1 , when no autocorrelation term is used, the best fit is obtained for a 3-regime 324 NHMM model $(\mathrm{BIC}=9873$, EVAR_train=90\% and EVAR_valid=85\%). When a first order 325 autocorrelation term is added, the best fit is issued from a 3-regime HMM-AR model: BIC=7997, 326 EVAR_train $=98 \%$ and EVAR_valid $=97 \%$. The lag-1 autocorrelation value is 0.85 for 327 EC_SPIM 1 , and therefore the weight given $\mathrm{Y}_{\mathrm{t}-1}$ is important compared to the other covariates, 328 EC_ $\mathrm{WH}_{1}$ and $\mathrm{EC}_{-} \mathrm{WND}_{2}$. This stresses the fact that when available first autoregressive term 329 should be included to enhance the performances 
The second mode of the EOF decomposition of the SPIM variability explains $7 \%$ of the total

331 variance. The selected predictors are the first mode of the Western wind, the tide, and the river

332 flow. The variability captured by EC_SPIM 2 relates to the local Westward wind, which is not

333 captured by the WH model, and the very coastal variability introduced by the tide and the river

334 outflow. For the non-AR models the selected model was the three-regime NHMM. It is interesting

335 to note in this case that EVAR_valid increased from 50 to $73 \%$ between the HMM and the NHMM,

336 highlighting the contribution of the non-homogeneous transition model. By contrast, the HMM-AR

337 performed slightly better than the NHMM-AR.

niveau de contribution des variables complémentaires

Table 1: Model performance for each EOF mode of the SPIM variability. For each

In bold are highlighted for each EC the selected configurations (see $\S 5.2$ ).

\begin{tabular}{|c|c|c|c|c|c|c|}
\hline \multirow{3}{*}{ EC_SPIM } & \multicolumn{7}{|c|}{ Number of modes, M } \\
\cline { 2 - 7 } & 1 & 2 & 3 & 1 & 2 & 3 \\
\hline \multirow{5}{*}{1} & HMM & HMM & HMM & HMM-AR & HMM-AR & HMM-AR \\
& (a) 11183 & 10037 & 9874 & 8157 & 7986 & $\mathbf{7 9 9 7}$ \\
& (b) 37 & 84 & 85 & 92 & 95 & $\mathbf{9 8}$ \\
& (c) 32 & 70 & 75 & 91 & 93 & $\mathbf{9 7}$ \\
\cline { 2 - 7 } & NHMM & NHMM & NHMM & NHMM-AR & NHMM-AR & NHMM-AR \\
& 11184 & 10037 & $\mathbf{9 8 7 3}$ & 8171 & 7994 & 8018 \\
& 37 & 84 & $\mathbf{9 0}$ & 92 & 92 & 98 \\
& 34 & 71 & $\mathbf{8 5}$ & 90 & 94 & 97 \\
\hline \multirow{5}{*}{2} & HMM & HMM & HMM & HMM-AR & HMM-AR & HMM-AR \\
& 9403 & 8579 & 8129 & 7167 & 7098 & $\mathbf{7 0 7 5}$ \\
& 18 & 67 & 76 & 90 & 91 & $\mathbf{9 2}$ \\
& 12 & 33 & 50 & 87 & 89 & $\mathbf{9 1}$ \\
\cline { 2 - 7 } & NHMM & NHMM & NHMM & NHMM-AR & NHMM-AR & NHMM-AR \\
& 9451 & 8614 & $\mathbf{8 1 5 2}$ & 7188 & 7383 & 7070 \\
& 18 & 67 & $\mathbf{7 9}$ & 89 & 90 & 92 \\
& 12 & 44 & $\mathbf{7 3}$ & 88 & 87 & 90 \\
\hline \multirow{5}{*}{3} & HMM & HMM & HMM & HMM-AR & HMM-AR & HMM-AR \\
& 8840 & 8222 & 7844 & 6723 & 6632 & $\mathbf{6 6 3 0}$ \\
& 12 & 57 & 68 & 85 & 86 & $\mathbf{8 8}$ \\
& 7 & 44 & 72 & 84 & 91 & $\mathbf{9 2}$ \\
\hline
\end{tabular}




\begin{tabular}{|c|c|c|c|c|c|c|}
\hline \multirow{5}{*}{} & NHMM & NHMM & NHMM & NHMM-AR & NHMM-AR & NHMM-AR \\
& 8866 & 8246 & $\mathbf{7 8 6 2}$ & 6745 & 6673 & 6703 \\
& 11 & 59 & $\mathbf{7 5}$ & 88 & 88 & 88 \\
& 16 & 45 & $\mathbf{7 6}$ & 86 & 91 & 92 \\
\hline \multirow{6}{*}{4} & HMM & HMM & HMM & HMM -AR & HMM -AR & HMM -AR \\
& 8248 & 7596 & 7285 & 6398 & 6416 & $\mathbf{6 3 1 3}$ \\
& 18 & 60 & 71 & 85 & 85 & $\mathbf{8 6}$ \\
& 28 & 63 & 72 & 86 & 86 & $\mathbf{8 6}$ \\
\cline { 2 - 7 } & NHMM & NHMM & NHMM & NHMM-AR & NHMM-AR & NHMM-AR \\
& 8276 & 7628 & $\mathbf{7 2 6 7}$ & 6426 & 6445 & 6357 \\
& 18 & 62 & $\mathbf{7 0}$ & 85 & 85 & 86 \\
& 28 & 59 & $\mathbf{7 5}$ & 83 & 83 & 85 \\
\hline
\end{tabular}

The third mode of the EOF decomposition of the SPIM variability explains $4 \%$ of the total variance. It captures some inter-annual and intra-seasonal variability of the latitudinal gradient of the SPIM. The selected predictors are EC_WH, $\mathrm{EC}_{-} \mathrm{WND} 1_{1}$ (Northern) and the tide. Once again, three-regime NHMM and HMM-AR provide the best results.

Regarding the fourth mode of the EOF decomposition of the SPIM variability, which accounts $3 \%$ of the total variance, EC_ $\mathrm{WH}_{1}, \mathrm{EC}_{-} \mathrm{WND} 2_{1}$, the tide and the river flow are selected as contributive predictors. We reconstruct $75 \%$ of EC_SPIM $\mathrm{S}_{3}$ variance of the validation dataset using a three-regime NHMM and $86 \%$ using a three-regime HMM-AR. We note that globally, the three indices (BIC, EVAR_train and EVAR_valid) tend to select the same models.

\subsection{Example with the estimation of EC_SPIM 1}

We report in Figure 5 the temporal evolution of the three regimes of the NHMM for EC_SPIM 1 . In table 2 are shown the corresponding coefficients for each predictor and the intercept. The first regime (light grey), characterized by high SPIM levels (intercept of 65), is referred as a 'winter regime'. The' winter regime' also strongly relates to the wave height (WH regression coefficient of 
360 2) identified as the 'summer regime'. For regimes 2 and 3, the coefficients for WH decrease 361 respectively to 0.12 and 0.09 . In summer the energy brought by waves is not sufficient enough to 362 re-suspend massively the sediments. It might be noticed that for all regimes the wind conditions 363 show a small but significant effect on EC_SPIM ${ }_{1}$. When an autocorrelation term is added (HMM364 AR, table 2), the $\mathrm{AR}(1)$ coefficient value is 0.86 for the regime 1 (winter), and 0.9 for regime 2 and 3653 which underlies the natural higher autocorrelation level of SPIM when the concentration is low.

Figure 4 compares the prediction of $\mathrm{EC}_{-} \mathrm{SPIM}_{1}$ using a single multivariate regression (green) and the proposed multi-regime NHMM. In this case the explained variance value (Table 1) is of $37 \%$ for the multivariate regression model compared to $85 \%$ for the three-regime NHMM.

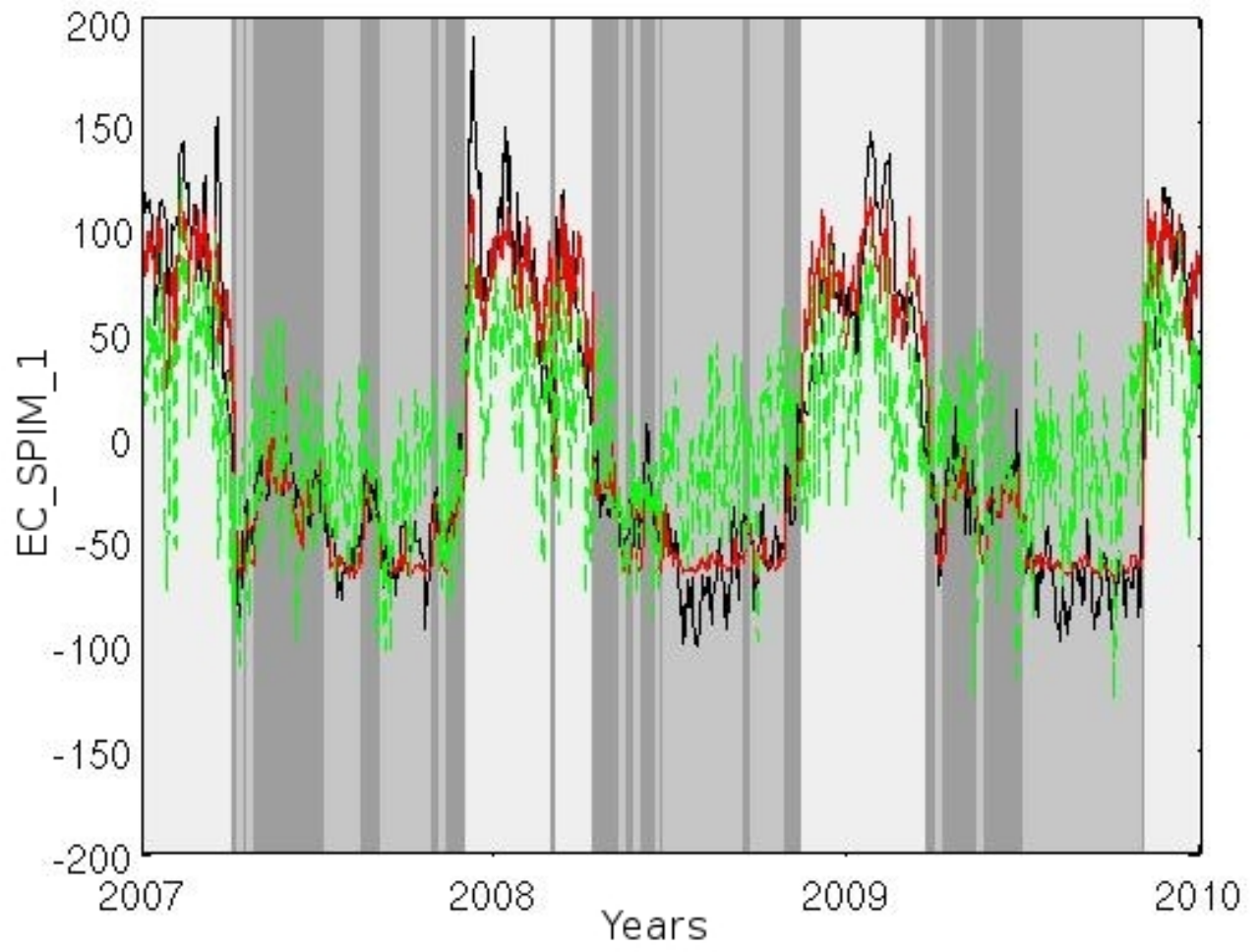

Figure 5: Estimation of the EC_SPIM 1 (in black) using EC_WH, $\mathrm{EC}_{-} \mathrm{WND} 2_{1}$ and a single regression (green) and a 3 regime NHMM (red). The nuances of grey in the background highlight the temporal distribution of the regimes. 
Table 2: Estimated regression parameters for each of the three regimes of the NHMM and the

HMM-AR for the first mode of the SPIM EOF decomposition: regression parameters involve an intercept and the regression coefficients of the wave height and western wind velocity.

\begin{tabular}{|c|c|c|c|c|}
\hline & $\mathrm{EC} \_\mathrm{WH}_{1}$ & $\mathrm{EC}_{-} \mathrm{WND}_{2}$ & Intercept & \\
\hline \multirow[t]{2}{*}{ NHMM } & $\begin{array}{l}(1, \text { winter }) 0.6037 \\
(2, \text { summer }) 0.0910 \\
(3, \text { transition }) 0.1210\end{array}$ & $\begin{array}{l}-0.0632 \\
0.0006 \\
0.0100\end{array}$ & $\begin{array}{l}65.0672 \\
-61.6442 \\
-24.6578\end{array}$ & \\
\hline & $\mathrm{EC} \mathrm{WH}_{1}$ & $\mathrm{EC} \quad \mathrm{WND}_{2}$ & Intercept & $\mathrm{AR}(1)$ \\
\hline HMM-AR & $\begin{array}{l}\text { (1) } 0.2383 \\
\text { (2) }-0.0050 \\
\text { (3) } 0.0354\end{array}$ & $\begin{array}{l}-0.0033 \\
0.0168 \\
0.0035\end{array}$ & $\begin{array}{l}4.4694 \\
-3.9531 \\
0.6079\end{array}$ & $\begin{array}{l}0.86 \\
0.90 \\
0.90\end{array}$ \\
\hline
\end{tabular}
$\left(Z_{t}=3\right)$ and 'winter' $\left(Z_{t}=1\right)$ regimes. The probability of switching from regime 3 to 1 increases with wave height covariate $\mathrm{WH}_{1}$ with a probability of switching close to zero when $\mathrm{WH}_{1}$ is negative and a probability close to one for large $\mathrm{WH}_{1}$ values.

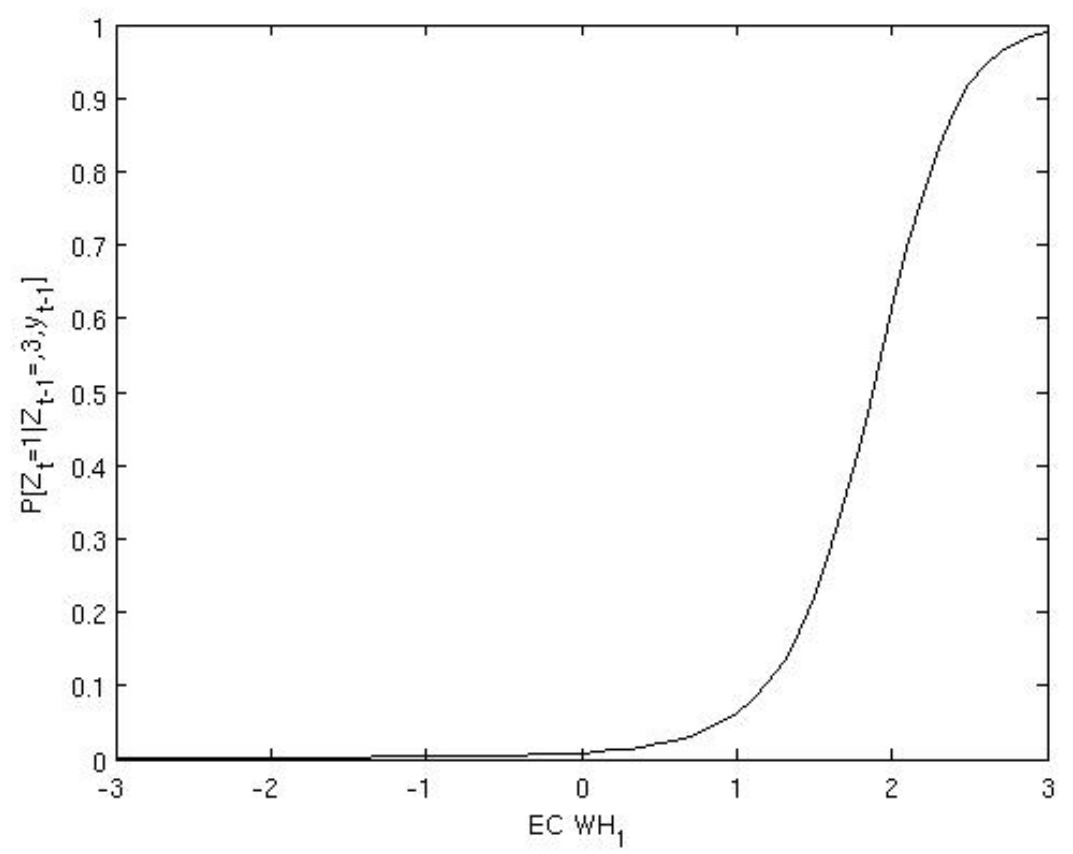

Figure 6: Non-homogeneous transition between 'transition regime' $(\mathrm{Zt}=3)$ (light grey Fig. 5)

384 and 'winter regime' $(\mathrm{Zt}=1)$ (light grey Fig. 5) as a function of the wave height covariate $\mathrm{WH}_{1}$. 


\subsection{Forcasting of the SPIM on the 2010 validation dataset}

We forecast SPIM fields from the reconstructed ECs and the selected models (Table $1 \&$ Eq. 19). Figure $7 \mathrm{a} \& 7 \mathrm{~b}$ compare EVAR_valid of the initial field (SPIM) using the three-regime NHMM and NHMM-AR models selected in Table 1 for their results. On average we were able to predict at $\mathrm{t}+180 \%$ of the variance using the NHMM (Fig. 7a) and $90 \%$ using the NHMM-AR. The spatial distribution of the error is not homogeneous. Fig. 7a shows that EVAR_valid value is of $90 \%$ in the Northern part with nevertheless poorer results in the South. Fig. $7 \mathrm{~b}$ shows that the $\mathrm{AR}_{1}$ component of the model increases EVAR for the whole area.
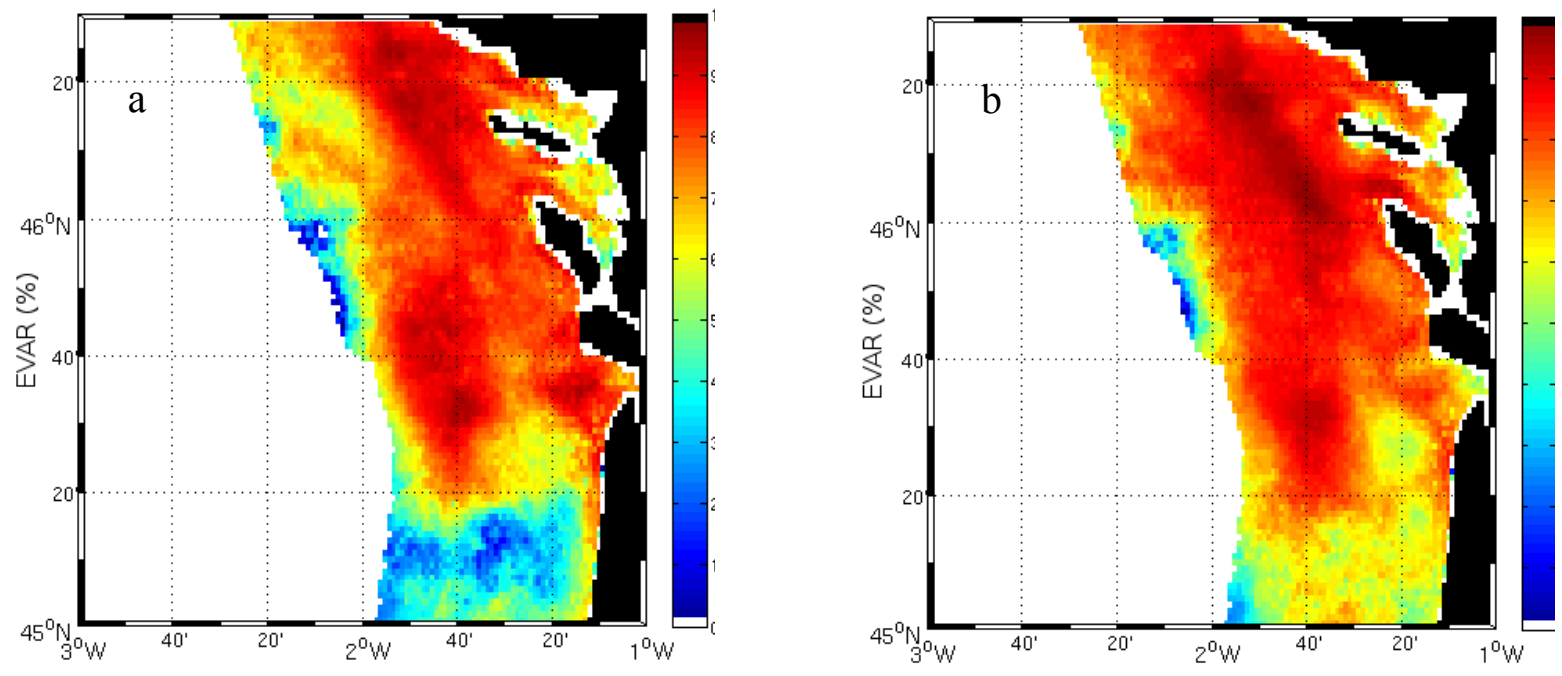

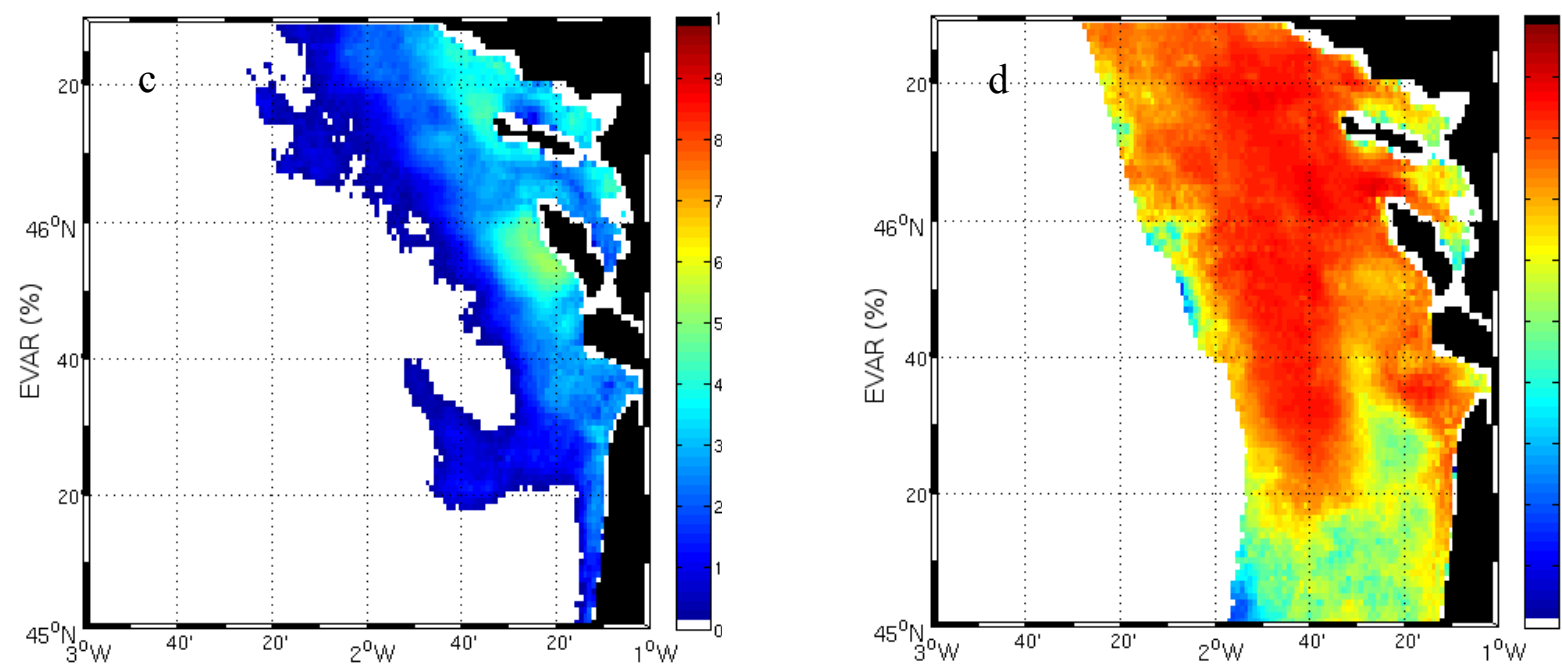

Figure 7: Explained variance for the 2010 validation dataset reconstructed using the selected 3regime NHMM (a) and NHMM-AR (b), compared with the standard multivariate regression without $\mathrm{AR}_{1}(\mathrm{c})$ and including an $\mathrm{AR}_{1}(\mathrm{~d})$.

To consider the model forecast performances, we report the short-term forecast results at different time steps (cf. Eq.13 \& 14). For the HMM-AR and NHMM-AR (Eq. 14) is the estimated value, the observation being not available (see $\S 3.2$ ). Table 3 synthesizes the explained variance statistics using 3 regimes and the four tested models for the forecasting at $t+1, t+5$ and $t+15$ and the 409 validation 2010 dataset (Eq. 19).

The long term forecasting results are globally better with the NHMM. At $t+15$ using the

411 NHMM we are able to forecast $74 \%$ of the variance for 2010 , compared with $40 \%$ for the HMM. In 412 this case where the covariates and predictors (mode outputs for which the short term predictions are 413 assumed to be available) are used in the estimation of the regime transition probability . For 414 autoregressive models, at $\mathrm{t}+15$, we were able to forecast $75 \%$ of the 2010 variance with the 415 NHMM-AR compared with 70\% with the HMM-AR. For the NHMM-AR the covariates help in the estimation of both and. At $\mathrm{t}+15$ NHMM and NHMM-AR show equivalent results underlying the maximal time-step to consider for the between these two models. 


\begin{tabular}{|c|c|}
\hline \multicolumn{2}{|c|}{ EVAR for the 2010 validation dataset } \\
\hline HMM & HMM-AR \\
$(\mathrm{t}+1) 73$ & 93 \\
$(\mathrm{t}+5) 63$ & 80 \\
$(\mathrm{t}+15) 40$ & 70 \\
& \\
& \\
NHMM & NHMM-AR \\
$(\mathrm{t}+1) 80$ & 93 \\
$(\mathrm{t}+5) 77$ & 82 \\
$(\mathrm{t}+15) 74$ & 75 \\
& \\
\hline
\end{tabular}

421 SVR model was also evaluated to evaluate the performances of a non-linear model on the studied 422 dataset. To perform the comparison, we train the SVR model (http://www.csie.ntu.edu.tw) for each 423 EC using the same training dataset (2007-2009) and performed forecasting using the same 424 validation dataset (2010). We used the setting as following: model epsilon-SVR ( $\mathrm{s}=3$ ), linear or 425 polynomial kernel ( $\mathrm{t}=0$ or 1 ) and the same inputs (predictors, covariables) for each EC. Parameters $426 \mathrm{c}$ and $\mathrm{g}$ [10] were optimised for each EC using the training dataset and the cross validation mode. 427 On the 2010 validation dataset, better forecast results reached $40 \%$ at $\mathrm{t}+1$ of the EVAR (without 428 AR) and $85 \%$ with an AR coefficient. The results were significantly worse than those obtained 429 using the time-varying models for increasing time steps. The SVR can address non-linear 430 relationships, it cannot nevertheless deal with multi-regime processes. By contrast, the latent-regime 431 model addresses by nature multi-regime processes and can approximate non-linear relationships as 432 a series of linear models. 


\section{Discussion}

HMM, NHMM, HMM-AR and NHMM-AR to characterize time-varying linear relationships between the high resolution inorganic suspended matter concentration (SPIM), estimated from 2007 to 2009 using MODIS, SeaWiFS and MERIS data in a coastal area, and its forcing conditions, i.e. the wave height, the Northern and Western winds, the tides and the river flow. The estimated 441 regimes are then used to forecast the SPIM using the independent year 2010 dataset, from $\mathrm{t}+1$ to $442 \mathrm{t}+15$.

An optimal number of three distinct geophysical regimes were needed to capture the different dynamics and optimize forecasting performance. Autoregressive and non-homogeneous model showed better performances. With the evaluated models and the 2010 validation dataset we were able to forecast at $\mathrm{t}+180 \%$ of the variance explained using a NHMM and $93 \%$ using a NHMMAR. In the latest case the strong natural autocorrelation of the studied signal is an important predictor to consider. The explained variance on the prediction at +1 for a standard multivariate regression was of $32 \%$ and $80 \%$ (with an $\mathrm{AR}_{1}$ term). Using a SVR we were able to forecast at $\mathrm{t}+1$ respectively $40 \%$ and $85 \%$ (with an $\mathrm{AR}_{1}$ ) of the explained variance.

As illustrated for the first SPIM EOF component (Figure 5), the proposed multi-regime setting allowed us to identify three seasonally varying relationships between the observed turbidity, the wave height and the wind. We did not drive the model to account for seasonal regimes but we identified three seasonally-discriminated regimes, with two leading factors: the mean SPIM level (intercept) and the Western wave height. These regimes identified directly physical behaviors, here the minimum of energy to be brought by the Western swell to re-suspend the sediments. This is regarded as a key feature of the latent-regime model compared to other non-linear regression models, such as Neural Networks [38] or SVR [10] which cannot address multi-regime 
459 relationships and are hardly interpreted in general. Using our dataset the non-linear SVR was not 460 able to retrieve the regime changes.

461 Regarding long-term forecast performance, at $\mathrm{t}+15$ best results obtained were of $74 \%$ of 462 explained variance for the NHMM and $75 \%$ for the NHMM-AR. For short period, typically from 1 463 to 15 days, when the observed $\mathrm{Y}$ is not available, NHMM-AR provided the best results. In this case 464 the predictors and covariates are used in the estimation of both and . At $t+15$ NHMM and NHMM465 AR showed similar results underlying the maximal time-step to consider, when no observation of $\mathrm{Y}$ 466 is available, for the choice between these two models.

In the future, we will address the forecasting of the chlorophyll-a using satellite-derived 468 observations such as the photosynthetic available radiation, the temperature, the suspended matters 469 (as index of available nutrients) and light attenuation [39]. In this more complicated case, second 470 order relationships between the variable and its predictors have to be evaluated, the chlorophyll-a 471 dynamic being not anymore a passive result of the forcing conditions, as expected with the SPIM, 472 but having its proper characteristics depending on each phytoplankton specie. Extensions of the 473 considered latent regime setting to other inverse problems in satellite sensing data analysis are also 474 under investigation, such as latent regime inversion procedures for satellite-derived chlorophyll-a 475 concentration to account for different water types (turbid or not turbid) and/or the presence of 476 specific phytoplankton species.

\section{$477 \quad 7$ Acknowledgements}

The authors thank Aldo Sottolichio from the Université of Bordeaux 1, for the provision of the 480 in-situ Gironde flow measurement, Pierre Tandeo for fruitful advises and the MCGS (Marine 481 Collaborative Ground Segment; http://www.mcgs.fr) project which aim at making the most of ESA 482 Sentinels satellites potential for users driven services based on high level products. MCGS 
483 addresses the need of the European Space Agency to build up data processing centers in 484 conjunction with the Copernicus Program for the provision of services to local and national, public 485 and private European institutions or entities involved in marine activities. The project is co-funded 486 by the French Government (Fonds Unique Inter-ministériel), local authorities of the Bretagne and 487 Provence-Alpes-Côte d'Azur regions and the European Regional Development Fund (ERDF), under 488 support of the French Space Agency (CNES).

\section{References}

1 P. Lazure, V. Garnier, F. Dumas, C. Herry, M. Chifflet. "Development of a hydrodynamic model of the Bay of Biscay”. Validation of hydrology. Continental Shelf Research, 29(8), 985-997, 2009.

2 L. Debreu, P. Marchesiello, P. Penven and G. Cambon, "Two-way nesting in split-explicit ocean models: algorithms, implementation and validation”, Ocean Model., 49-50, 1-21, 2012.

3 A. Sottolichio, P. Le Hir, P. Castaing. "Modeling mechanisms for the turbidity maximum stability in the Gironde estuary, France”, Coastal and Estuarine Fine Sediment Processes, pp 373-386, 2000.

4 A. Rivier, F. Gohin, P. Bryere, C. Petus, N. Guillou, G. Chapalain. "Observed vs. predicted variability in non-algal suspended particulate matter concentration in the English Channel in relation to tides and waves”. Geomarine Letters, 32(2), 139-151, 2012.

5 C. Aitken, "On Least Squares and Linear Combinations of Observations", Proceedings of the Royal Society of Edinburgh, 55, 42-48, 1935. Spline Smoothing Journal of The American Statistical Association , 78(381):81-89, 1983. 
8 Tesfaye, Y. G., Meerschaert, M. M., and Anderson, P. L. Identification of periodic autoregressive moving average models and their application to the modeling of river flows. Water Resources Research, 42(1), 2006

9 Some Neural Network applications in environmental sciences part I: forward and inverse problems in geophysical remote measurements, V. Krasnopolsky and H. Schiller, Neural Networks, vol 16 (2003), 321334.

10 Chih-Chung C., Chih-Jen L. LIBSVM: A library for support vector machines Transactions on Intelligent Systems and Technology (TIST), 2011.

11 P. Ailliot, V. Monbet. "Markov-switching autoregressive models for wind time series". Environmental Modelling \& Software, 30, 92-101, 2012.

12 F. Gohin, S. Loyer, M. Lunven, C. Labry, J.M. Froidefond, D . Delmas, M . Huret, A. Herbland, "Satellite-derived parameters for biological modelling in coastal waters: illustration over the eastern continental shelf of the Bay of Biscay”. Remote Sensing of Environnement 95(1), 29-46, 2005.

13 D. Doxaran, « Télédétection et modélisation numérique des flux sédimentaires dans l'estuaire de la Gironde », PhD thesis, University Bordeaux 1, France, 326 pp, 2002.

14 D. Doxaran, J.M. Froidefond, P. Castaing and M. Babin. "Dynamics of the turbidity maximum zone in a macrotidal estuary (the Gironde, France): Observations from field and MODIS satellite data", Estuarine Coastal and Shelf Science, 81, 321-332, 2009.

15 D.G. Bowers, C.E. Binding.”The optical properties of mineral suspended particles: a review and synthesis". Estuarine Coastal Shelf Science, 67(1/2), 219-230, 2006.

16 D. Eisma, P. Bernhard, G.C. Cadée, V. Ittekkot, J. Kalf, R. Laane, J.M. Martin, W.G. Mook, A. Van Put, T. Schuhmacher, "Suspended matter particle size in some West-European estuaries; part II: a review on floc formation and break up”, Netherlands Journal of Sea Research, 28(3), 215-220, 1991. 
18 P. Tandeo, B. Chapron, S. Ba, E. Autret, R. Fablet, "Segmentation of Mesoscale Ocean Surface

Dynamics Using Satellite SST and SSH Observations Geoscience and Remote Sensing", IEEE Transactions on Volume, pp 1-9, 2013.

19 B.H. Juang, and L.R. Rabiner. "Hidden Markov models for speech recognition”, Technometrics, 33, 251-272, 1991.

20 C. Frankignoul and K. Hasselmann, "Stochastic climate models. Part II: Application to SST anomalies and thermocline variability". Tellus, 29, 289-305, 1977.

21 R.W. Preisendorfer, "Principal Component Analysis in Meteorology and Oceanography", Elsevier, New York,. pp 425, 1988.

23 Abdi, H. "Discriminant correspondence analysis." In: N.J. Salkind (Ed.): Encyclopedia of Measurement and Statistic. Thousand Oaks (CA): Sage. pp. 270-275, 2007. waves. Part I: Definition, calibration, and validation”. Journal of Physical Oceanography, 40(9):1917-1941, 2010.

“Gridded

Surface

Wind Journal of Remote Sensing, 33: $549 \quad$ 1729-1754, 2011. Océanographique de la Marine, Brest, Rapport 564-UJA, 2000 
29 J. P. Hughes and P. Guttorp, "Incorporating Spatial Dependence and Atmospheric Data in a Model of Precipitation”. Journal Applied Meteorology, 33, 1503-1515, $1994 \mathrm{~b}$.

30 A.P. Dempster, N.M. Laird and D.B. Rubin, "Maximum likelihood from incomplete data via the EM algorithm", Journal of the Royal Statistical Society. Series B (Methodological), 39, 1-38, 1977. Wind

32 W. Zucchini and P. Guttorp. "A hidden Markov model for space-time precipitation", Water Resources Research, 27, 1917-1923, 1991. York, 2005.

34 H.S. Bhat, N. Kumar, "On the derivation of the Bayesian Information Criterion", 2010, Available from http://nscs00.ucmerced.edu/ nkumar4/BhatKumarBIC.pdf. MODIS/Aqua, and SeaWiFS chlorophyll-a data from 1998 to 2008 on the European Atlantic shelf”, IEEE Trans Geoscience Remote Sensing 49(1), 143-154, 2011. linear trends in multisensor time series in the presence of autocorrelated noise: Application to the chlorophyll-a SeaWiFS and MERIS data sets and extrapolation to the incoming Sentinel 3-OLCI mission”, Journal of Geophysical Research Oceans, 118, 3752-3763, 2013.

38 H. Schiller, R. Doerffer, "Neural network for emulation of an inverse model operational derivation 1999. 
$578 \quad 39$ B. Saulquin, A. Hamdi, F. Gohin, J. Populus, A. Mangin, O. Fanton D'Andon, "Estimation of the 579 diffuse attenuation coefficient K-dPAR using MERIS and application to seabed habitat mapping”. Remote 580 Sensing Of Environment, 128, 224-40 